\title{
Dynamic Material Parameters in Molecular Dynamics and Hydrodynamic Simulations on Ultrashort-Pulse Laser Ablation of Aluminum
}

\author{
Stefan Scharring, Marco Patrizio, Hans-Albert Eckel, Johannes Roth, \\ and Mikhail Povarnitsyn
}

\begin{abstract}
Molecular dynamics reveals a detailed insight into the material processes. Among various available codes, IMD features an implementation of the twotemperature model for laser-matter interaction. Reliable simulations, however, are restricted to the femtosecond regime, since a constant absorptivity is assumed. For picosecond pulses, changes of the dielectric permittivity $\epsilon$ and the electron thermal conductivity $\kappa_{e}$ due to temperature, density and mean charge have to be considered. Therefore, IMD algorithms were modified for the dynamic recalculation of $\epsilon$ and $\kappa_{e}$ for every timestep following the corresponding implementation in the hydrodynamic code Polly-2T. The usage of dynamic permittivity yields an enhanced absorptivity during the pulse leading to greater material heating. In contrast, increasing conductivity induces material cooling which in turn decreases absorptivity and heating resulting in a higher ablation threshold. This underlines the importance of a dynamic model for $\epsilon$ and $\kappa_{e}$ with longer pulses which is commonly often neglected. Summarizing all simulations with respect to absorbed laser fluence, ablation depths in Polly-2T are two times higher than in IMD. This can be ascribed to the higher spallation strength in IMD stemming from the materialspecific potential deviating from the equations of state used in Polly-2T.
\end{abstract}

\footnotetext{
S. Scharring $\bullet$ H.-A. Eckel

German Aerospace Center (DLR), Institute of Technical Physics, Stuttgart, Germany

e-mail: stefan.scharring@dlr.de; hans-albert.eckel@dlr.de

M. Patrizio

Technical University of Darmstadt, Institute for Nuclear Physics, Darmstadt, Germany e-mail: m.patrizio@gsi.de
}

J. Roth $(\triangle)$

Institute of Functional Materials and Quantum Technologies (FMQ), University of Stuttgart,

Stuttgart, Germany

e-mail: johannes.roth@fmq.uni-stuttgart.de

M. Povarnitsyn

Research Center for High Energy Density Physics, Joint Institute for High Temperatures (JIHT),

Russian Academy of Sciences (RAS), Moscow, Russia

e-mail: povar@ihed.ras.ru 


\section{Introduction}

Among the variety of available open-source codes for the simulation of laser ablation, Polly-2T from the Joint Institute of High Temperatures at the Russian Academy of Sciences, Moscow, and IMD from the Institute of Functional Materials and Quantum Technologies at the University of Stuttgart are very different simulation tools which can partially attributed to their strongly varying numerical approaches, namely hydrodynamics (HD) in the case of Polly-2T and molecular dynamics (MD) for IMD. A first comparison between both programs was given in [1] revealing similar results for a restricted parameter range as well as large discrepancies beyond this range.

Some differences between both codes, however, stem from the unequal implementation of the physics of laser-matter interaction in both programs. Numerical work with IMD was mainly focusing on so-called cold laser ablation with fs laser pulses where material changes during laser irradiation are less relevant than with long pulses. With increasing pulse length, however, the interaction of matter and laser beam becomes more and more apparent, since optical permittivity, electron thermal conductivity as well as thermal coupling from the electron gas to the ion lattice depend both on density and temperature. Since large gradients of $\varrho, T_{e}$ and $T_{i}$ might occur during a laser pulse, absorption of laser energy is subject to significant changes due to the laser-induced temporal and spatial fluctuations of temperature and density.

Models that cover a wide parameter range of parameter range of $\varrho, T_{e}$ and $T_{i}$ are implemented in Polly-2T take into account for these effects. Their implementation into IMD is the scope of this paper in order to enable a better comparison of simulation results from both codes.

\section{Theoretical Background}

\subsection{Two-Temperature Model}

Energy deposition by an ultrafast laser pulse into a metal target can be described by the two-temperature Model (TTM) [2] by

$$
\begin{gathered}
c_{e}\left(T_{e}\right) \frac{\partial T_{e}}{\partial t}=\nabla\left[\kappa_{e}\left(T_{e}\right) \nabla T_{e}\right]-\gamma_{e i}\left(T_{e}-T_{i}\right)+S(\mathbf{r}, t) \\
c_{i}\left(T_{i}\right) \frac{\partial T_{i}}{\partial t}=\nabla\left[\kappa_{i}\left(T_{i}\right) \nabla T_{i}\right]+\gamma_{e i}\left(T_{e}-T_{i}\right) .
\end{gathered}
$$

where the indices e and i denote the electron and ionic subsystem, resp., $c$ is the specific heat capacity, $T$ the temperature, $\kappa$ represents the thermal conductivity, $\gamma_{e i}$ 
is the electron-phonon coupling parameter, and $S(\mathbf{r}, t)$ is the spatial and temporal distribution of the energy density. For ultrashort laser pulses, $\kappa_{i}$ can be neglected [3] as it is done in Polly-2T and IMD.

\subsection{Wide-Range Models}

In the wide-range models treated in this paper it is assumed that the transition of the material from the metal phase into the plasma phase occurs in the vicinity of the Fermi temperature $T_{F}$. Under this assumption, material transport properties can be written as an interpolation between the behavior in the metal phase and the behavior in the plasma phase as sketched in the following subsections $[4,5]$.

\subsubsection{Optical Transport Properties}

The electric permittivity $\epsilon$ of the target material is described by a temperaturedependent interpolation of $\epsilon_{\mathrm{met}}$ in the metal phase and $\epsilon_{\mathrm{pl}}$ in the plasma phase

$$
\epsilon=\epsilon_{\mathrm{pl}}+\left(\epsilon_{\mathrm{met}}-\epsilon_{\mathrm{pl}}\right) \cdot e^{-A_{\mathrm{opl}} \cdot T_{e} / T_{F}}
$$

where $A_{\text {opt }}$ is an empirical parameter adjusted to match experimental values. $\epsilon_{\text {met }}$ is composed of the band-to-band contribution $\epsilon_{\mathrm{bb}}$ which is taken from tabulated data and an intraband term

$$
\epsilon_{\mathrm{met}}\left(\omega_{L}, \varrho, T_{i}, T_{e}\right)=\epsilon_{\mathrm{bb}}+1-\frac{n_{e}}{n_{c r}\left(1+i v_{\mathrm{eff}, \mathrm{opt}} / \omega_{L}\right)}
$$

where $\omega_{L}$ is the frequency of the laser, $\varrho$ is the density of the material, $n_{\mathrm{cr}}$ is the critical concentration of electrons and the effective collision frequency $v_{\text {eff,opt }}$ as derived in detail in [4].

In the plasma phase, for temperatures far above $T_{F}, \epsilon_{\mathrm{pl}}$ can be calculated as

$$
\epsilon_{\mathrm{pl}}\left(\omega_{L}, \varrho, T_{e}\right)=1-\frac{n_{e}}{n_{\mathrm{cr}}} K\left(\frac{\nu_{\mathrm{pl}}}{\omega_{L}}\right)
$$

where $K\left(\frac{v_{\mathrm{pl}}}{\omega_{L}}\right)$ is an empirical function.

\subsubsection{Heat Transport Properties}

Similar to Eq. (3), the thermal conductivity $\kappa_{e}$ of the electron gas can be written as an interpolation from the metal phase to the plasma phase according to

$$
\kappa_{e}=\kappa_{\mathrm{pl}}+\left(\kappa_{\mathrm{met}}-\kappa_{\mathrm{pl}}\right) \cdot e^{-A_{\mathrm{he}} \cdot T_{e} / T_{F}}
$$


where $A_{\text {he }}$ is an empirical parameter adjusted to match experimental values. In the metal phase $\kappa_{\text {met }}$ can be calculated according to the Drude formalism as

$$
\kappa_{\text {met }}\left(\varrho, T_{i}, T_{e}\right)=\frac{\pi^{2} k_{B}^{2} n_{e}}{3 m_{e} v_{\text {eff,he }}\left(T_{i}, T_{e}\right)} T_{e}
$$

where $k_{B}$ is Boltzmann's constant, $n_{e}$ is the electron concentration, $m_{e}$ the electron mass and $\nu_{\text {eff,he }}$ the effective collision frequency as shown in [5].

For the plasma phase $\kappa_{\mathrm{pl}}$ can be calculated as

$$
\kappa_{\mathrm{pl}}=\frac{16 \sqrt{2} k_{B}\left(k_{B} T_{e}\right)^{5 / 2}}{\pi^{3 / 2} Z e^{4} \sqrt{m_{e}} \Lambda}
$$

where $Z$ is the mean charge of the ions, $e$ is the elementary charge and $\Lambda$ is the Coulomb logarithm.

Accordingly, thermal coupling from the electron gas to the ion lattice can be described in a wide-range approach by

$$
\gamma_{e i}\left(\varrho, T_{i}, T_{e}\right)=\frac{3 k_{B} m_{e}}{m_{i}} n_{e} v_{\mathrm{eff}, \mathrm{ei}}\left(T_{i}, T_{e}\right)
$$

where $v_{\text {eff,ei }}$ represents the corresponding collision frequency.

\section{Numerical Codes}

\subsection{Hydrodynamic Simulations}

\subsubsection{Target Material}

The thermodynamic material properties are treated in Polly-2T using semi-empirical two-temperature multiphase equations of state (EOS) comprising the Thomas-Fermi description of thermal effects of the electron gas. The EOS provide for tabulated functions for pressure $P_{e}\left(\varrho, T_{e}\right), P_{i}\left(\varrho, T_{i}\right)$ and specific energy $e_{e}\left(\varrho, T_{e}\right), e_{i}\left(\varrho, T_{i}\right)$ of both subsystems which allow for the solution of the TTM equations. From the EOS, the equilibrium mean charge of ions $\langle Z\rangle$ can be derived as well.

The phase diagram of aluminum corresponding to the EOS is shown in Fig. 1.

\subsubsection{Two-Temperature Model}

A sound description of the specific simulation assumptions in Polly-2T is given in [4]. Laser-matter interaction is described here by the TTM in a single-fluid 1D 


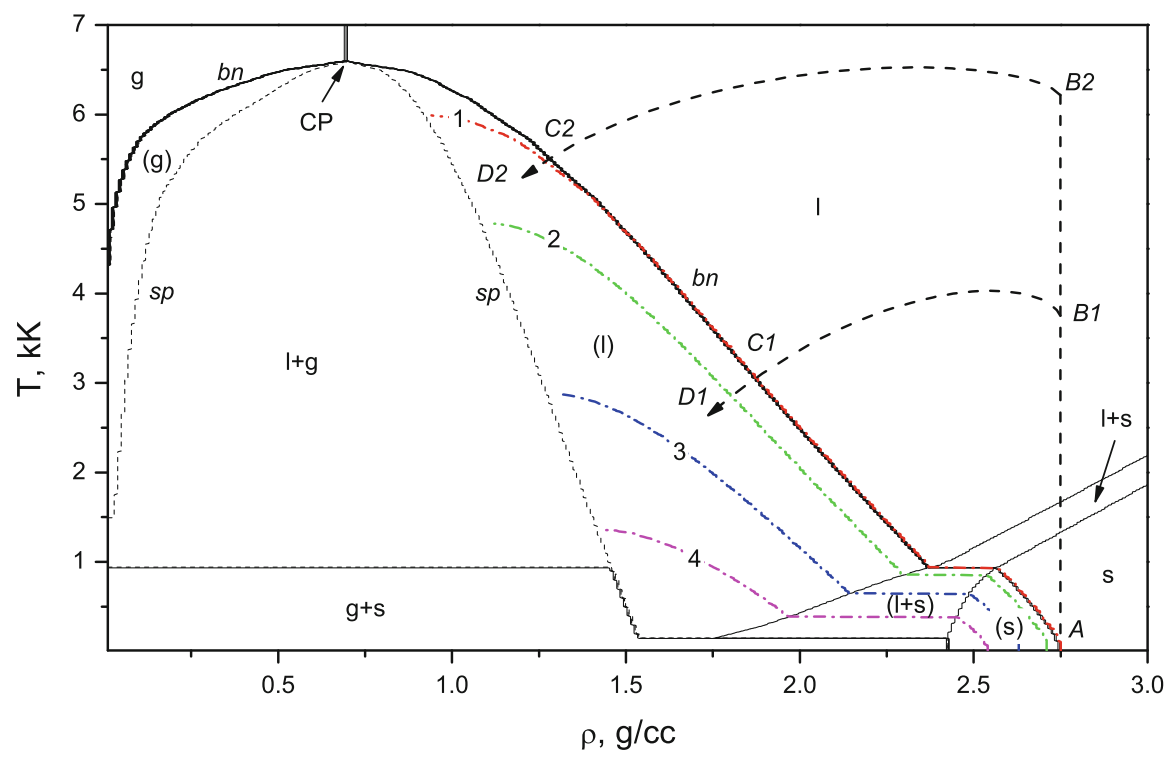

Fig. 1 Phase diagram of aluminum according to the EOS used in Polly-2T, taken from [6]. Abbreviations: sp: spinodal; bn: binodal; g: stable gas; 1: stable liquid; s: stable solid; $1+$ s: stable melting; $1+\mathrm{g}$ : liquid-gas mixture; $\mathrm{g}+\mathrm{s}$ : sublimation zone; (g): metastable gas; (1): metastable liquid; $(1+\mathrm{s})$ : metastable melting; (s): metastable solid; CP: critical point. Dash-dot lines: isobars; 1: $0 \mathrm{GPa} ; 2:-1 \mathrm{GPa} ; 3:-3 \mathrm{GPa} ; 4:-5 \mathrm{GPa}$

Lagrangian form yielding a modification of Eqs. (1) and (2) by

$$
\begin{gathered}
\frac{\partial e_{e}}{\partial t}+P_{e} \frac{\partial v}{\partial m}=\frac{\partial}{\partial m}\left[\varrho \kappa_{e} \frac{\partial T_{e}}{\partial m}\right]-\gamma_{e i}\left(T_{e}-T_{i}\right) / \varrho+S(x, t) / \varrho \\
\frac{\partial e_{i}}{\partial t}+P_{i} \frac{\partial v}{\partial m}=+\gamma_{e i}\left(T_{e}-T_{i}\right) / \varrho
\end{gathered}
$$

where $e_{e}$ and $e_{i}$ denote the specific energy of the electrons and ions, resp., $P$ is the pressure, $m$ mass, $\varrho$ density, $v$ velocity, and, in contrast to [4], radiation transport phenomena are neglected here. Conservation of mass and energy are granted using

$$
\begin{gathered}
\frac{\partial(1 / \varrho)}{\partial t}-\frac{\partial v}{\partial m}=0, \\
\frac{\partial v}{\partial t}+\frac{\partial\left(P_{i}+P_{e}\right)}{\partial m}=0 .
\end{gathered}
$$




\subsubsection{Transport Properties}

In the present version of Polly-2T, which is accessible online [7] for web-based simulations as well, the wide-range models for $\epsilon, \kappa_{e}$, and $\gamma_{e i}$ are implemented. With respect to thermal transport properties, this means that the dependencies $\kappa_{e}\left(\varrho, T_{i}, T_{e}\right)$ and $\gamma_{e i}\left(\varrho, T_{i}, T_{e}\right)$, cf. Eqs. (6) and (9) are considered in the solver for Eqs. (10)-(13).

With respect to the permittivity $\epsilon$, the Helmholtz equation is solved for the source term $S(x, t)$ in Eq. (10) considering the spatial and temporal fluctuations of the permittivity due to the dependencies given by $\epsilon\left(\omega_{L}, \varrho, T_{i}, T_{e}\right)$, cf. Eq. (3).

\subsection{Molecular Dynamics Simulations}

\subsubsection{Target Material}

Particle interactions in IMD were represented using the embedded atom model (EAM) from Ercolessi and Adams [8]. The potential takes into account the interactions between an atom and its closest neighbors while introducing a cut-off distance for longer ranges, thus creating a multi-body problem that can only be solved numerically. For any given atom inside such a system the potential can be described as

$$
V_{i}=\frac{1}{2} \sum_{i, j, i \neq j} \phi\left(r_{i j}\right)+F\left[\sum_{j} \varrho_{e}\left(r_{i j}\right)\right]
$$

where the EAM potential $\phi$ contains the interaction between the cores of atoms i and $\mathrm{j}$ and the EAM embedding function $F$ describes the interaction of the electron hull of atom i with all its neighbors while influenced by the local electron density $\varrho_{e}\left(r_{i j}\right)$.

The phase diagram of aluminum corresponding to the EAM potential is shown in Fig. 2.

\subsubsection{Two-Temperature Model}

Whereas heat transport in the ion lattice can be described by the multi-particle interactions using the EAM potential, a supplementary system had to be added to the MD simulations to take into account energy absorption and propagation inside the electron gas. Hence, solving Eq. (1) with a Finite-Difference (FD) scheme, hybrid simulations are carried out in IMD, where electron-phonon coupling from the electronic FD subsystem into the MD core is realized by a dynamic coupling 


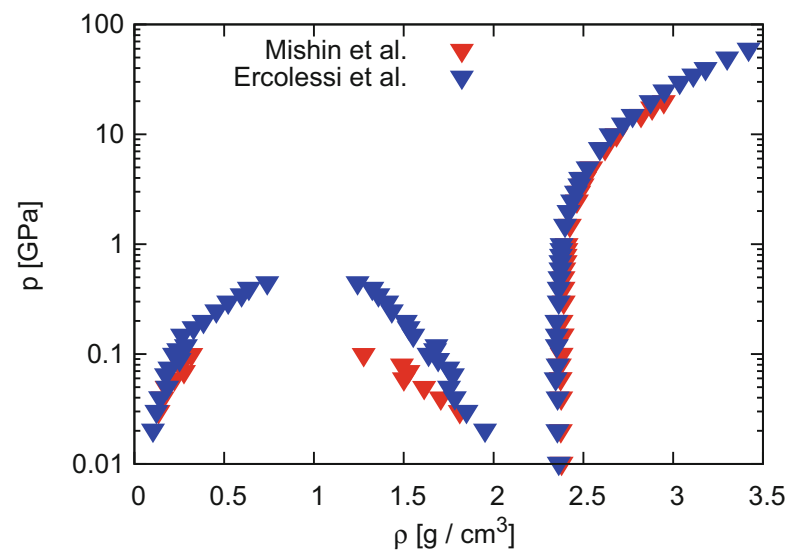

Fig. 2 Phase diagram of aluminum according to the EAM potential data from Ercolessi [8], and Mishin [9], resp.

parameter $\xi$ following

$$
m_{i} \frac{\partial^{2} \mathbf{r}_{i}}{\partial t^{2}}=-\nabla V_{i}+\xi \cdot m_{i} \mathbf{v}_{T, i}
$$

with

$$
\xi=\frac{\frac{1}{n} \sum_{k=1}^{n} \gamma_{e i} V_{N}\left(T_{e, k}-T\right)}{\sum_{j} m_{j}\left(v_{T, j}\right)^{2}}
$$

where $v_{T, i}=v_{i}-v_{\mathrm{CMS}}$ denotes the thermal velocity of the $i$ th atom taking into account for the velocity of the center of mass (CMS) of the corresponding FD cell.

\subsubsection{Transport Properties}

Since applications of IMD have mainly been focused on cold ablation, i.e., laser pulses in the femtosecond regime, in the open-source version of IMD dynamic material parameters were only implemented partially, in contrast to Polly-2T. The electron heat capacity is modeled using $c_{e}=T_{e} \cdot 135 \mathrm{~J} /\left(\mathrm{m}^{3} \mathrm{~K}^{2}\right)$ which is rather good comparable to Polly-2T where $P_{e}\left(\varrho, T_{e}\right)$ and $e_{e}\left(\varrho, T_{e}\right)$, given by the EOS data, yield $c_{e} \approx T_{e} \cdot 100 \mathrm{~J} /\left(\mathrm{m}^{3} \mathrm{~K}^{2}\right)$. Electronic heat conductivity and coupling coefficient, however, are treated as constants in IMD using $\kappa_{e}=235 \mathrm{~J} /(\mathrm{s} \mathrm{m} \mathrm{K})$ and $\gamma_{e i}=5.69 \cdot 10^{17} \mathrm{~J} /\left(\mathrm{s} \mathrm{m}^{3} \mathrm{~K}\right)$, resp., for aluminum.

In the original version of IMD, absorption of laser energy is described by the Beer-Lambert law requiring the amount of absorbed energy as input for the MD simulation. 


\section{Numerical Work}

\subsection{Code Developments}

The focus of our work on IMD was the stepwise implementation of the wide-range models for $\epsilon\left(\omega_{L}, \varrho, T_{i}, T_{e}\right), \kappa_{e}\left(\varrho, T_{i}, T_{e}\right)$ and $\gamma_{e i}\left(\varrho, T_{i}, T_{e}\right)$ described in Sect. 2.2. For a better comparison of MD and HD results, wide-range models of Polly-2T were switched off if not active in IMD, i.e., the upgrades of IMD were accompanied by corresponding downgrades of Polly-2T. A detailed description of the code developments can be found in [10].

In the first step, denoted as cold reflectivity, we assumed fixed material constants and described the optical properties of aluminum by an optical absorption coefficient of $\alpha=1.267 \cdot 10^{8} \mathrm{~cm}^{-1}$ corresponding to an optical penetration depth of $l_{\alpha}=$ $\alpha^{-1}=7.89 \mathrm{~nm}$ and a surface reflectivity of $R=0.94331$. Hence, in our simulations with this version of IMD, the absorbed fluence $\Phi_{\mathrm{abs}}=R \cdot \Phi$ was taken as input parameter for the absorbed laser fluence and $\alpha$ was used for energy allocation following Lambert-Beer. For convenience, an interface was implemented in IMD for the direct input of $\Phi$ calculating $R$ and $\Phi_{\text {abs }}$ depending on incidence angle and polarization of the laser beam. Accordingly, the corresponding Polly-2T downgrade simulations employed a complex refractive index of $n=1.74+10.72 \cdot i$ as input parameter. Moreover, in those runs electron thermal conductivity and electronphonon coupling coefficient were set fixed to $\kappa_{e}=235 \mathrm{~W} / \mathrm{K} \mathrm{m}$ and $\gamma_{e i}=5.69$. $10^{17} \mathrm{~W} /\left(\mathrm{K} \mathrm{m}^{3}\right)$, resp.

In the second step, the wide-range model for $\epsilon$ was implemented in IMD and re-activated in Polly-2T. Whereas in Polly-2T absorption of laser energy is implemented using the Helmholtz equation, $\alpha$ was calculated for each FD cell in IMD for every time step allowing for a dynamic allocation of absorbed energy following Lambert-Beer.

Finally, the wide-range model for $\kappa_{e}$ has been implemented in IMD and reactivated in Polly-2T in addition to the wide-range model for $\epsilon$. The implementation of the wide-range model for $\gamma_{e i}$ in IMD, however, has not been finished successfully yet at present.

\subsection{Simulation Setup}

For the incident laser pulse, the temporal course of the intensity is given by

$$
I(t)=I_{0} \cdot \exp \left[-0.5 \cdot\left(t / \sigma_{t}\right)^{2}\right]
$$

with

$$
I_{0}=\Phi /\left(2.507 \cdot \sigma_{t} \cdot \cos \vartheta\right)
$$


where $\Phi$ is the incident laser fluence and $\vartheta$ is the incidence angle. In the following, we denote the pulse length $\tau$ the full width half maximum (FWHM) with $\tau$ R $=$ $\hat{\mathrm{R}} \tau_{\mathrm{FWHM}}=2 \sqrt{2 \ln 2} \cdot \sigma_{t}$. Simulations were started approximately at the point in time $t_{\text {in }}$ before the laser pulse where $I\left(t_{\text {in }}\right)=0.1 \mathrm{~W} / \mathrm{cm}^{2}$. With respect to our related work on laser micropropulsion [11], $\lambda=1064 \mathrm{~nm}$ as laser wavelength and, for the sake of simplicity, an incidence angle of $\vartheta=0^{\circ}$ with linear polarized light was chosen. Pulse lengths of $\tau \approx 50 \mathrm{fs}, 500 \mathrm{ps}$, and $5 \mathrm{ps}$, resp., were chosen in combination with fluences of $\Phi \approx 0.19,0.37,0.74,1.49$, and $2.49 \mathrm{~J} / \mathrm{cm}^{2}$, resp.

Since the dimension of the simulation cells in Polly-2T is in the nanometer range and therefore rather large, it is not disadvantageous with respect to the computational effort to create bulk material with a certain thickness, which was chosen as $1 \mathrm{~mm}$ here. The shock wave stemming from the ablation event is supposed to travel with the corresponding speed of sound and will have no impact on the rear side of the target within the simulation time $t_{\text {sim. }}$.

In contrast to HD simulations, computational time scales linearly with the number of particles in IMD. Therefore, samples with a thickness of $650 \mathrm{~nm}$ have been created which is sufficient for a time span of $\approx 100 \mathrm{ps}$ after the laser pulses for shock wave to travel to the rear side of the sample [1]. $20 \mathrm{~nm}$ have been chosen as lateral extension of the sample using periodic boundary conditions [12].

\section{Results}

\subsection{Energy Conservation}

Simulations results with the original version of IMD are shown in Fig. 3. The laser pulse energy is absorbed by the electron gas and is coupled into the ion lattice.

A comparison of the additional system energy with the absorbed laser fluence, however, shows, that in several cases the target appears to acquire more energy than delivered from the laser pulse, cf. Fig. 4. Even long after the laser pulse, the energy in the electron subsystem still increases. This unphysical behavior was already observed in [1] and can be ascribed to numerical errors in the FD computation describing the electron system in IMD since this phenomenon is especially pronounced for large laser fluences in conjunction with very short laser pulses. Code upgrades have been implemented at the FMQ yielding a significant improvement for low fluences, cf. Fig. 4. For greater fluences, however, the system energy is still divergent, albeit at a lower level, whereas for very high fluences, i.e., $\Phi_{\text {in }}=2.97 \mathrm{~J} / \mathrm{cm}^{2}$ in the most cases, simulations were aborted after computation errors.

Analysis of the system energy comprising the wide-range models for $\epsilon$ and $\kappa_{e}$ reveals that an absorbed fluence of roughly $\Phi_{\mathrm{abs}} \approx 0.1 \mathrm{~J} / \mathrm{cm}^{2}$ seems to be the upper limit for reasonable system behavior in IMD simulations, cf. Fig. 5 . Correspondingly, simulations with $\Phi_{\mathrm{abs}} \geq 0.1 \mathrm{~J} / \mathrm{cm}^{2}$ are discarded in the following. 


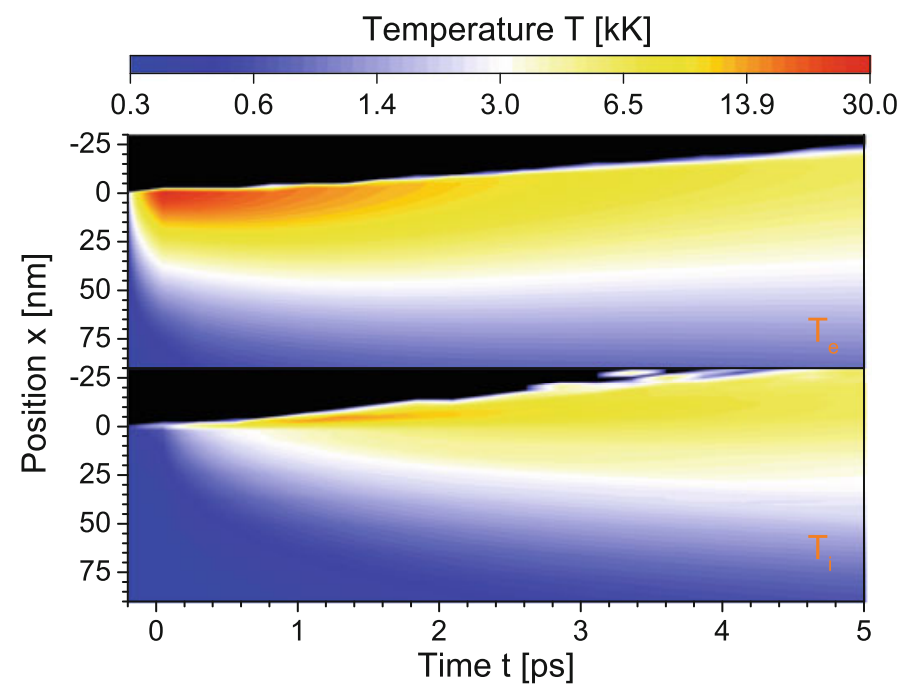

Fig. 3 Temporal and spatial evolution of the electron temperature $T_{e}$ and lattice temperature $T_{i}$, results from IMD with constant $\epsilon, \kappa_{e}$. Target material: aluminum, $\tau=50 \mathrm{fs}, \Phi=0.74 \mathrm{~J} / \mathrm{cm}^{2}$

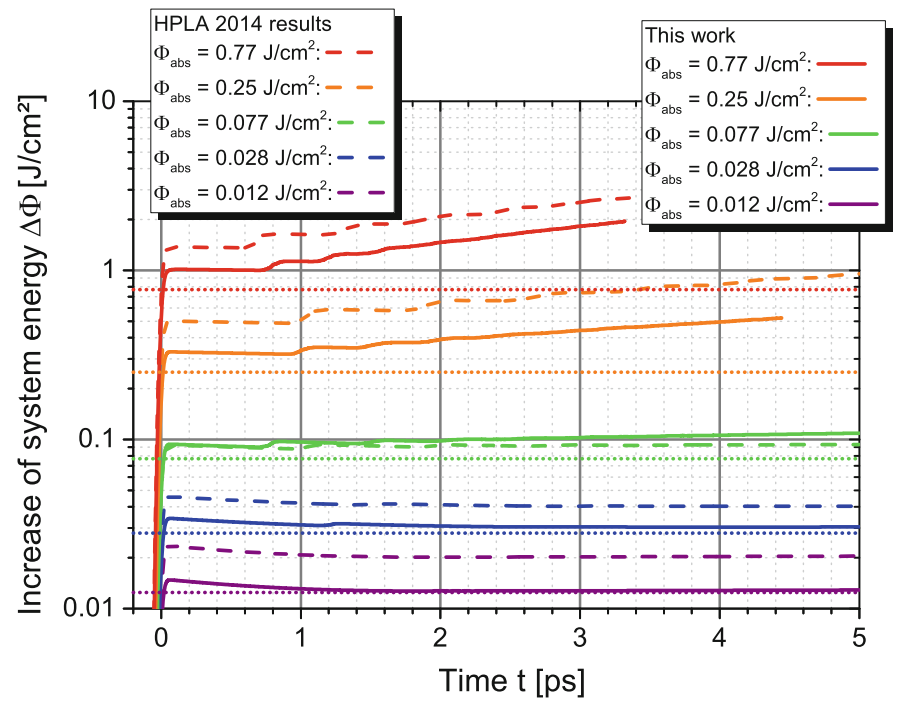

Fig. 4 Temporal course of additional system energy for various laser fluences at $\tau=50 \mathrm{fs}$. The absorbed laser fluence $\Phi_{\text {abs }}$ which is an input parameter of IMD is compared with the actual increase of system energy due to the laser pulse. Earlier results refer to the simulations shown at the HPLA 2014 [1] whereas results from this work denote simulations with the present status of IMD prior the upgrades treated in this paper 


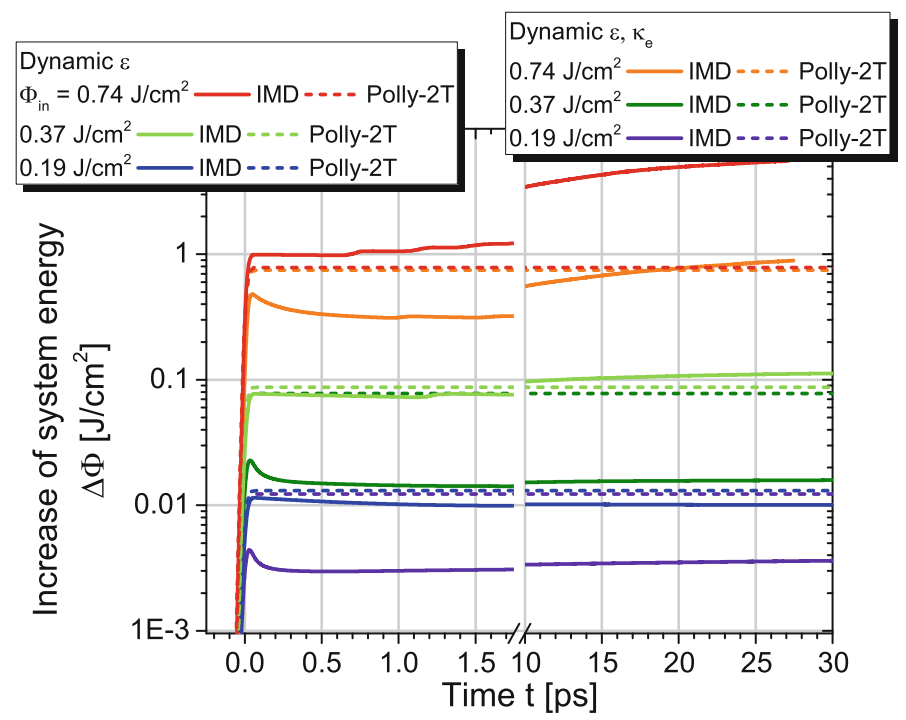

Fig. 5 Temporal course of the system energy during and after laser absorption. For higher fluences, a significantly large increase is found with IMD simulations. Results from hydrodynamic simulations with Polly-2T are given for comparison (dashed lines)

In any case, the course of the system energy shows an overshoot after the laser pulse, followed by a minimum and a slow drift upwards. This drift is rather pronounced for high fluences.

With Polly-2T which has much more coarse spatial resolution such large energy drifts have not been observed in the given laser parameter range but at fluences being one order of magnitude higher and beyond.

\subsection{Permittivity}

For very short laser pulses with moderate intensities, absorption of laser energy in the cold reflectivity case is comparable to the findings with implementation of the wide-range model for the permittivity $\epsilon$, cf. Fig. 6. This might be associated with cold ablation and deduced to the thermal confinement of the system due to the relaxation time $\tau_{e}$ of the electron gas which prevents a significant change of the target properties during the laser pulse.

The picture turns, however, for higher fluences and/or pulse lengths which can be found most clearly in the results from HD simulations with Polly-2T, cf. Fig. 7. In this case, a deeper and faster heat dissipation in the electron gas can be detected comparing Fig. 3 with Fig. 8. This can be attributed to the temperature dependency 


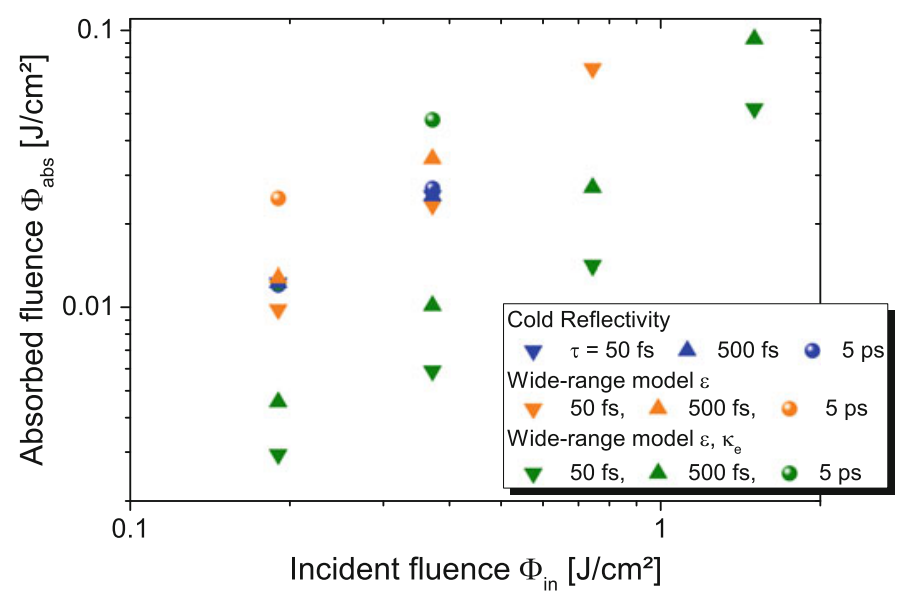

Fig. 6 Absorbed fluence vs. incident fluence, results from IMD for various upgrade steps

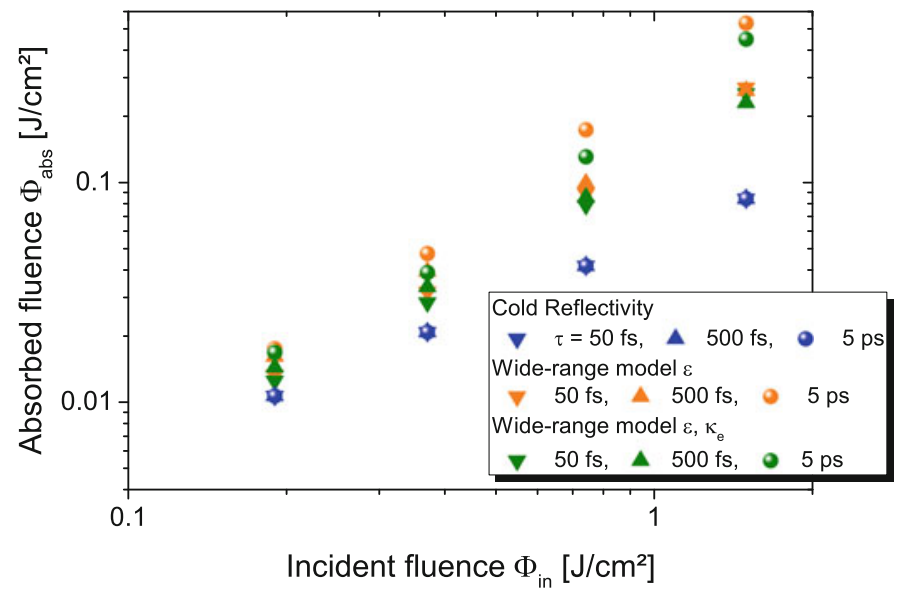

Fig. 7 Absorbed fluence vs. incident fluence, results from Polly-2T for various downgrade steps

of $\epsilon$ that is apparent in the strong changes of the refractive index which yield a higher optical penetration depth in the heat affected zone, cf. Fig. 9.

At the transition from target to vacuum, with $l_{\alpha} \approx 36 \mathrm{~nm}$ a rather high value for the penetration depth is found. This can be ascribed to the relatively large size of the FD-cells. They comprise 4 MD-cells containing both vacuum and the surface layers of the solid target. Hence, a rather low density is calculated for the FD-cell leading to a low absorption coefficient in the respective cell following the widerange model for the permittivity. On the whole, however, this can be regarded as a residual error with negligible impact on the simulation results which applies as well 


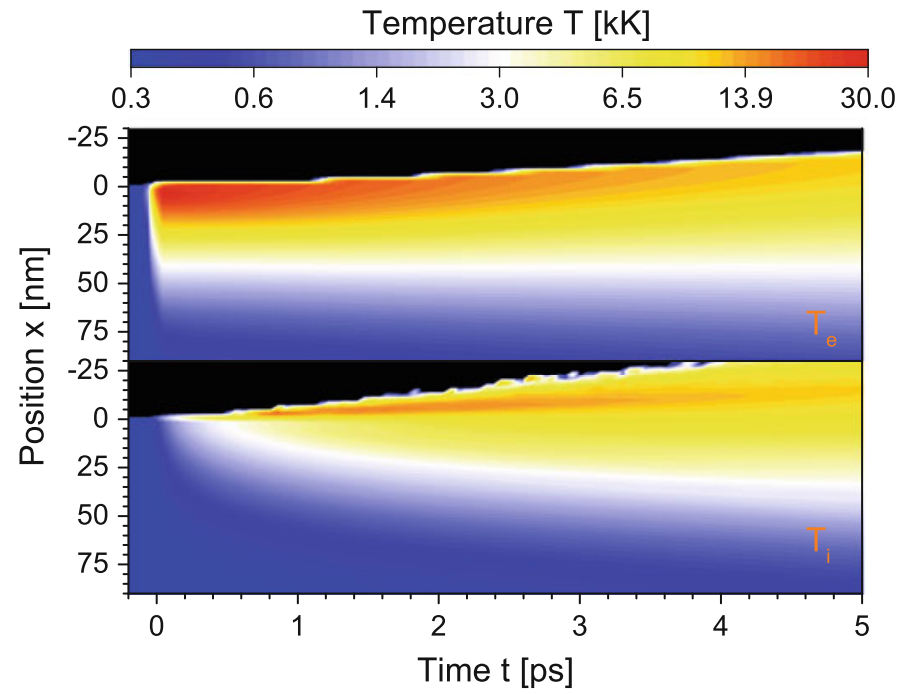

Fig. 8 Temporal and spatial evolution of the electron temperature $T_{e}$, results from IMD with $\epsilon=\epsilon\left(\varrho, T_{e}, T_{i}\right)$ according to the wide range model. Target material: aluminum, $\tau=50 \mathrm{fs}$, $\Phi=0.74 \mathrm{~J} / \mathrm{cm}^{2}$

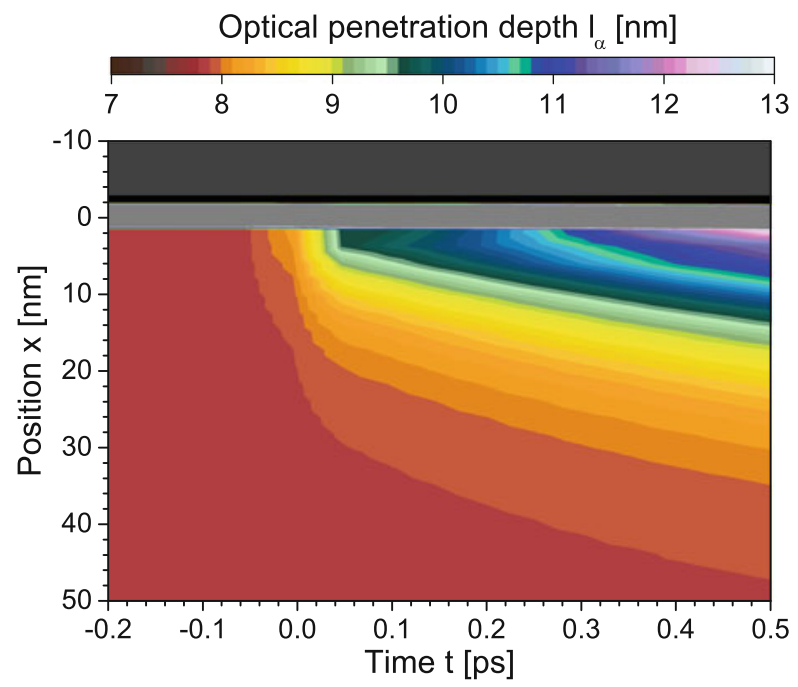

Fig. 9 Temporal and spatial evolution of the absorption length $l_{\alpha}$, results from IMD with $\epsilon=\epsilon\left(\varrho, T_{e}, T_{i}\right)$ according to the wide range model. Target material: aluminum, $\tau=50 \mathrm{fs}$, $\Phi=0.74 \mathrm{~J} / \mathrm{cm}^{2}$ 


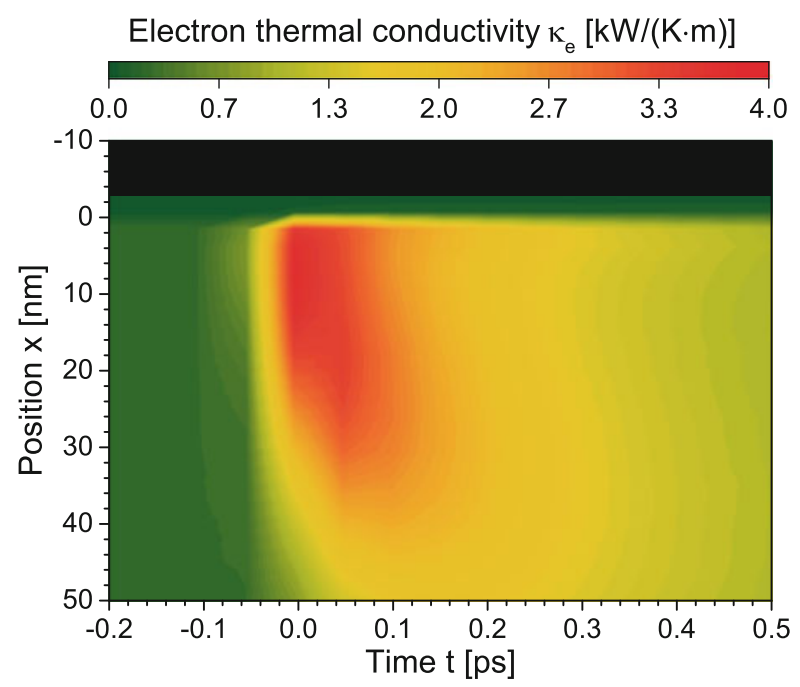

Fig. 10 Temporal and spatial evolution of the electronic heat conductivity $\kappa_{e}$, results from IMD with $\epsilon=\epsilon\left(\varrho, T_{e}, T_{i}\right)$ and $\kappa=\kappa\left(\varrho, T_{e}, T_{i}\right)$ according to the wide range model. Target material: aluminum, $\tau=50 \mathrm{fs}, \Phi=0.74 \mathrm{~J} / \mathrm{cm}^{2}$

for the calculations with the wide-range model on the electron thermal conductivity, cf. Fig. 10.

\subsection{Electron Thermal Conductivity}

When the electron gas is heated during laser pulse, the electron thermal conductivity raises according to the wide-range model. For the chosen example, an increase by the order of one magnitude is found at the surface layer of the target, cf. Fig. 10.

Due to the greater thermal conductivity $\kappa_{e}$ the laser energy is dissipated into deeper layers of the material, cf. Fig. 11 in comparison with Fig. 8. Therefore, heating of the material surface as well as material expansion are less pronounced than under the assumption of constant $\kappa_{e}$. In turn, changes in the permittivity are rather low compared to the previous case where the wide-range model was only implemented for $\epsilon$. Hence, the overall absorbed energy is significantly reduced as can be seen from Fig. 6.

Whereas this decrease of absorbed energy due to the dynamic behavior of $\kappa_{e}$ is very pronounced in IMD simulations, this trend is much weaker for the results from HD simulations with Polly-2T, cf. Fig. 7. The reason for this large discrepancy is not quite clear. Since $\kappa_{e}$ directly affects the FD scheme for the electron gas, the above-mentioned problems with the unphysical increase of energy in the electron subsystem for high fluences might have a deeper reason that is mirrored here as well. 


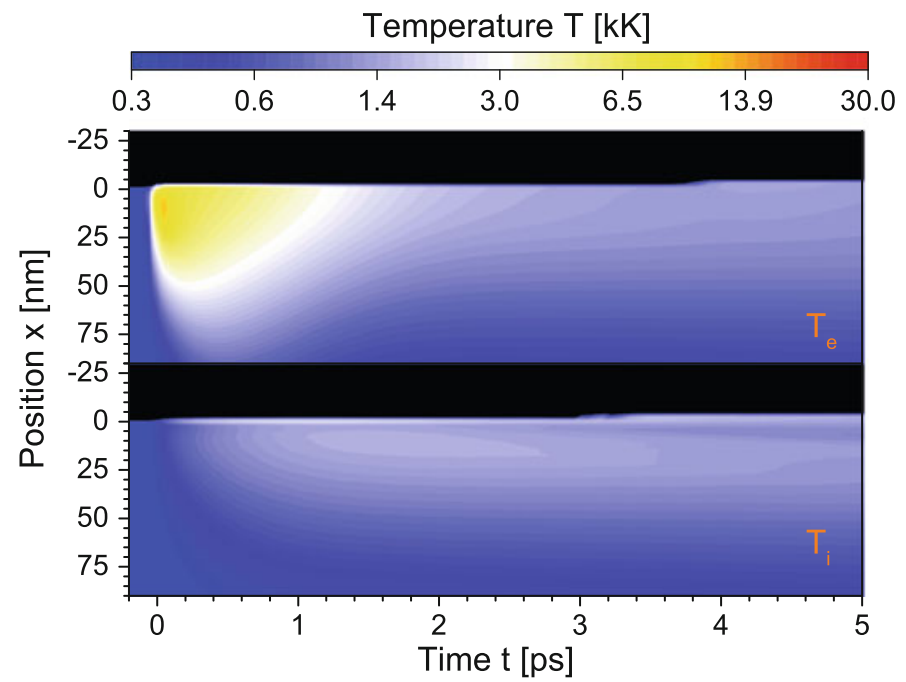

Fig. 11 Temporal and spatial evolution of the electron temperature $T_{e}$, results from IMD with $\epsilon=$ $\epsilon\left(\varrho, T_{e}, T_{i}\right)$ and $\kappa=\kappa\left(\varrho, T_{e}, T_{i}\right)$ according to the wide range model. Target material: aluminum, $\tau=50 \mathrm{fs}, \Phi=0.74 \mathrm{~J} / \mathrm{cm}^{2}$

\subsection{Ablation}

It is evident that large deviations in absorbed energy, as shown in Figs. 6 and 7, yield a different behavior of the target material. Therefore, it is meaningful to have a look at the laser-induced processes with respect to the absorbed fluence, irrespective of the incident fluence, cf. Fig. 12. Whereas it is straightforward to determine $\Phi_{a b s}$ for the Polly-2T simulations, for IMD $\Phi_{a b s}$ has to be assessed from its temporal course, cf. Fig. 5. As an approximation, the point in time where the minimum of $\Delta \Phi$ occurs has been taken in order to minimize the impact of overshoot and drift. For the $5 \mathrm{ps}$ pulses, however, drift is apparently present already during the laser pulse, hence, $-t_{\text {in }}$ has been chosen instead.

It can be seen that in the most cases more material is ablated in the hydrodynamic simulations than in the MD case. Correspondingly, the ablation threshold in Polly$2 \mathrm{~T}$ is lower than in IMD, as can be seen as well from the data in Table 1. Nevertheless, all simulation data are roughly in the range of experimental results.

When the amount of ablated mass in HD and MD simulations is compared, the different models for materials have to be taken into account. Apart from issues related to the wide-range model of $\epsilon, \kappa_{e}$ and $\gamma_{e i}$, underlying EOS in Polly-2T and EAM potential in IMD, resp., imply different spall strengths for the target material which is $2 \mathrm{GPa}$ for Polly-2T and $8.7 \mathrm{GPa}$ for IMD, where the latter value is taken from the EAM potential of Mishin [9] being rather similar the one of Ercolessi and Adams [8] used in the present MD simulations. The higher spall strength in IMD is 


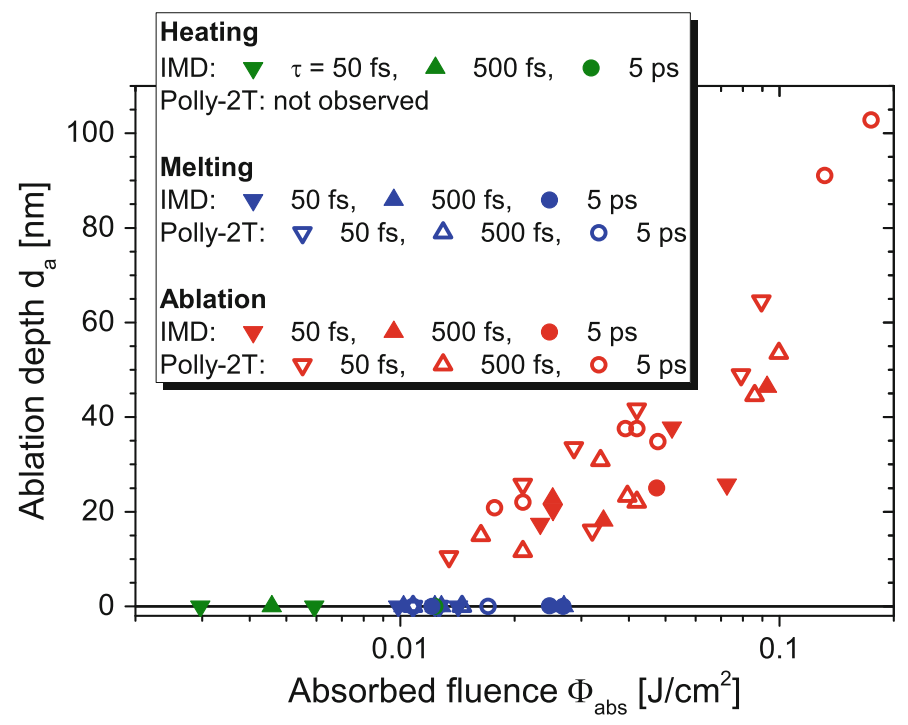

Fig. 12 Ablation depth $d_{a}$ and laser-induced processes in dependence of the absorbed laser fluence $\Phi_{\text {abs }}$ for molecular dynamics simulations with IMD in comparison with results from hydrodynamic simulations with Polly-2T comprising all steps of implementation of the wide-range models of $\epsilon$ and $\kappa_{e}$

Table 1 Threshold $\Phi_{0}$ for laser ablation of aluminum, results from hydrodynamic simulations with Polly-2T as well as IMD molecular dynamics simulations in comparison with own experimental results [16] and literature data

\begin{tabular}{l|l|l|l}
\hline$\tau(\mathrm{ps})$ & $\lambda(\mathrm{nm})$ & $\Phi_{0}\left(\mathrm{~J} / \mathrm{cm}^{2}\right)$ & Reference \\
\hline 0.05 & 1064 & $0.3^{c},<0.2^{\epsilon}, 0.3^{\epsilon, \kappa_{e}}, 0.6^{\epsilon, \kappa_{e}, \gamma_{e i}}$ & Polly-2T \\
\hline 0.05 & 1064 & $0.2^{c}, 0.3^{\epsilon}, 0.7^{\epsilon, K_{e}}$ & IMD \\
\hline 0.1 & 1240 & 0.7 & {$[13]$} \\
\hline 0.13 & 800 & 0.034 & {$[14]$} \\
\hline 0.5 & 1064 & $0.3^{c},<0.2^{\epsilon}, 0.3^{\epsilon, K_{e}}, 0.4^{\epsilon, K_{e}, \gamma_{e i}}$ & Polly-2T \\
\hline 0.5 & 1064 & $0.2^{c}, 0.2^{\epsilon}, 0.7^{\epsilon, K_{e}}$ & IMD \\
\hline 0.5 & 1054 & $\approx 0.05$ & {$[15]$} \\
\hline 5 & 1064 & $0.3^{c},<0.2^{\epsilon}, 0.3^{\epsilon, K_{e}}, 0.2^{\epsilon, K_{e}, \gamma_{e i}}$ & Polly-2T \\
\hline 5 & 1064 & $0.3^{c}, 0.2^{\epsilon}, 0.3^{\epsilon, K_{e}}$ & IMD \\
\hline 10 & 1064 & 0.4 & {$[16]$} \\
\hline
\end{tabular}

Indices refer to the level of implementation of the wide-range models (c: cold reflectivity)

presumably the reason for the typically lower mass removal and the higher ablation threshold in IMD compared with results from hydrodynamic simulations.

Ionization is taken into account in Polly-2T in the calculation of the cell's specific energy, but neglected in the EAM potential of IMD. This is supposed to have no significant impact on the ablated mass but rather on the velocity of the ablation jet where kinetic energy might be overestimated in IMD [1]. In the calculation of permittivity according to the wide-range model, however, ionization is implicitly 
taken into account for both IMD and Polly-2T being calculated from electron density via the electron temperature.

\section{Conclusion and Outlook}

Simulations of laser-matter interaction during an ultrashort laser pulse have been performed evaluating the usage of wide-range models for permittivity $\epsilon$ and electron thermal conductivity $\kappa_{e}$ as strongly temperature-dependent material parameters.

Since the absorbed fluence mainly rules the laser-induced processes in the investigated cases for $\tau=50$ and $500 \mathrm{fs}$, substitution of the wide-range models by the input of the effectively absorbed energy, as shown recently [1], can be a suitable workaround. For $\tau=5 \mathrm{ps}$, i.e., longer pulse lengths in the range of the electronphonon coupling time $\tau_{i}$, however, the dynamic issue of laser-matter-interaction comes into play and this approach does not hold any more.

Future work with IMD comprising the wide-range models requires parallelization of the upgraded code. This is the main restriction up to now, whereas the standard version is already parallelized by default.

Taking into account for dynamic material properties for a wide range of temperatures and density in laser-matter-interaction, both codes a principally wellsuited for the current work of our group on laser-ablative micropropulsion [11].

3D simulations of surface roughness under multiple spot ablation are needed there for the assessment of corresponding thrust noise. Such simulations are basically feasible in IMD, albeit at a much lower spatial scale, as well as with Polly$2 \mathrm{~T}$ with a quasi-2D approach neglecting lateral side-effects [17].

However, the mentioned problems in IMD with higher fluences limit its practical use in this field of application since the anticipated working point for the future microthruster is in the range of at least 3 to 6 times of the ablation threshold [18].

For future work, implementation of laser-induced ablation comprising widerange models presented here into a commercial finite-element method (FEM) software is planned.

\section{References}

1. S. Scharring, D.J. Foerster, H.-A. Eckel, J. Roth, M. Povarnitsyn, Open access tools for the simulation of ultrashort-pulse laser ablation, in High Power Laser Ablation/Beamed Energy Propulsion (2014). Available via DLR. http://elib.dlr.de/89090/

2. S.I. Anisimov, B.L. Kapeliovich, T.L. Perel'man, Electron emission from metal surfaces exposed to ultrashort laser pulses. Ann. Mat. Pura Appl. 39, 375-377 (1974)

3. D. Baeuerle, Laser Processing and Chemistry (Springer, Berlin, 2000)

4. M.E. Povarnitsyn, N.E. Andreev, P.R. Levashov, K.V. Khishchenko, O.N. Rosmej, Dynamics of thin metal foils irradiated by moderate-contrast high-intensity laser beams. Phys. Plasmas 19, 023110 (2012) 
5. M.E. Povarnitsyn, N.E. Andreev, E.M. Apfelbaum, T.E. Itina, K.V. Khishchenko, O.F. Kostenko, P.R. Levashov, M.E. Veysman, A wide-range model for simulation of pump-probe experiments with metals. Appl. Surf. Sci. 258, 9480-9483 (2012)

6. M.E. Povarnitsyn, K.V. Khishchenko, P.R. Levashov, Phase transitions in femtosecond laser ablation. Appl. Surf. Sci. 255, 5120-5124 (2009)

7. Virtual Laser Laboratory (VLL). Joint Institute for High Temperatures (JIHT), Russian Academy of Sciences (RAS). (2012). http://vll.ihed.ras.ru/

8. F. Ercolessi, J.B. Adams, Interatomic potentials from first-principles calculations: the forcematching method. Europhys. Lett. 26, 583-588 (1994)

9. Y. Mishin, D. Farkas, M.J. Mehl, D.A. Papaconstantopoulos, Interatomic potentials for monoatomic metals from experimental data and ab initio calculations. Phys. Rev. B 59, 3393 3407 (1999)

10. M. Patrizio, Upgrade of the IMD software tool for the simulation of laser-matter interaction. Technical University of Darmstadt (2015). Available via DLR. http://elib.dlr.de/100164/

11. S. Scharring, S. Karg, R.-A. Lorbeer, N., Dahms, H.-A. Eckel, Low-noise thrust generation by laser-ablative micropropulsion, in 34th International Electric Propulsion Conference, Kobe, Paper 2015-143 (2015). Available via DLR. http://elib.dlr.de/97576/

12. D.J. Foerster, Validation of the software package IMD for molecular dynamics simulations of laser induced ablation for micro propulsion. University of Stuttgart (2013). Available via DLR. http://elib.dlr.de/83975/

13. S.I. Anisimov, N.A. Inogamov, Y.V. Petrov, V.A. Khokhlov, V.V. Zhakhovskii, K. Nishihara, M.B. Agranat, S.I. Ashitkov, P.S. Komarov, Thresholds for front-side ablation and rear-side spallation of metal foil irradiated by femtosecond laser pulse. Appl. Phys. A 92, 797-801 (2008)

14. C. Guo, Structural phase transition of aluminum induced by electronic excitation. Phys. Rev. Lett. 84, 4493-4496 (2000)

15. K. Kremeyer, J. Lapeyre, S. Hamann, Compact and robust laser impulse measurement device, with ultrashort pulse laser ablation results. AIP Conf. Proc. 997, 147-157 (2008)

16. L. Pastuschka, Optimization of material removal for laser-ablative microthrusters, Master thesis, University of Stuttgart, 2015

17. S. Scharring, R.-A. Lorbeer, S. Karg, L. Pastuschka, D.J. Foerster, H.-A. Eckel, The MICROLAS concept: precise thrust generation in the Micronewton range by laser ablation. IAA Book Ser. Small Satell. 6, 27-34 (2016)

18. S. Scharring, R.-A. Lorbeer, H.-A. Eckel, Numerical simulations on laser-ablative micropropulsion with short and ultrashort laser pulses. Trans. JSASS Aerospace Tech. Jpn. 14, Pb 69-Pb 75 (2016) 